\title{
Novel Technique (Woven Darning Method) in the Management of Large Midline Incisional Hernias
}

\author{
Prof. N. Tamilselvan ${ }^{1}$ M.S., Dr. M.Rajasekar ${ }^{2}$ M.S., Dr. S.S. Meera ${ }^{3}$ M.S., \\ ${ }^{1,2,3}$ (Associate Professor of General Surgery, Government Mohan Kumaramangalam Medical College, \\ Salem, Tamilnadu, India)
}

\begin{abstract}
Ventral hernia repair has been a challenge to the surgeons from time immemorial. To do a tension free repair is our goal and prevention of recurrence is our aim especially in incisional hernias with large defect. Many techniques like Anatomical repair, Keel's repair and Mayo's double breasting etc. have been developed. These techniques are not adequate in the management of midline incisional hernias with large defects. Abrahamson developed a technique of creation of a new linea alba using the anterior rectus sheath and a Shoelace like polypropylene darning to reinforce the gaps at the lateral cut edge of the rectus sheath. After that a modification was advocated by fixing a polypropylene mesh to the lateral cut edges. Still the theoretical possibility of the gaps is there, moreover there is enormous fibrous tissue reaction with mesh contracture along with the chance of infection. With this background in mind, we advocate a novel technique of reinforcing the rectus sheath gap with a scaffold of two layered polypropylene darning in a woven pattern. In this technique, the usage of large quantity of foreign material (polypropylene mesh) is avoided. At the same time, adequate reinforcement is obtained with no recurrence so far, in our study
\end{abstract}

Keywords: Woven darning, Shoelace repair, Midline Incisional hernias

\section{Introduction}

Ventral hernia repair has been a challenge to the surgeons from time immemorial. To do a tension free repair is our goal and prevention of recurrence is our ultimate aim especially in incisional hernias with large defect. Many techniques like Anatomical repair, Keel's repair and Mayo's double breasting technique, etc have been developed. Though all these techniques are very good and time tested with excellent results they are unable to fulfill the expectations in the management of large midline incisional hernias with a huge defect between the two recti. For a better outcome in these cases Abrahamson developed a technique of creation of a new linea alba using the anterior rectus sheath and he used a Shoelace like polypropylene darning to reinforce the muscles and the fascia that are either week or atrophied. But even then there existed gaps at the lateral cut edges with a possibility of hernia recurrence. Hence a modification of this technique was advocated by fixing a polypropylene mesh to the lateral cut edges of the rectus sheath to bridge these gaps. In this technique, the mesh is just fixed to the edges of the rectus sheath but doesn't overlap, hence these gaps still persist more over there is enormous fibrous tissue reaction with mesh contracture, and also the chance of mesh infection is high. With this background in mind, we conceived a novel technique of reinforcing the gap in the rectus sheath by a scaffold of two layered polypropylene darning with a woven pattern, which offers a very good result in our limited follow up period. At the same time the usage of large quantity of foreign material (polypropylene mesh) can be avoided with less chance of infection.

\section{Aims and Objectives}

To study the efficacy and outcome of the novel technique of Woven Darning method in the management of large midline incisional hernias.

\section{Materials and Methods}

3.1. Study design: Prospective Non-Randomized study.

3.2. Study Group: Over one year, 20 patients with large midline incisional hernias in Surgery department of GMK Medical college hospital, Salem were enrolled in our study. There were 12 males with 8 females with age preponderance of 15 to 70 years. The average age was 35 years with previous history of surgery followed by wound infection and wound gaping.

3.3. Inclusion Criteria:

1. Male and female with large midline incisional hernias

2. Age between 15 and 70 years.

3.4. Exclusion Criteria:

1. Other hernias except Post Laparotomy Incisional hernias

2. Hernias with features of Obstruction and Strangulation. 
3. Age less than 15 and more than 70 .

4. Co morbid hepatic, renal pathologies, diabetes and Coronary Heart disease.

\section{Novel Technique Instituted}

In our technique, hernial defect is approached through a midline vertical incision (after excising the previous scar in appropriate cases) and the contents were reduced back into peritoneal cavity. Since the sac was a single layer with peritoneum and the rectus sheath fused together peritoneum cannot be separated alone in most of the cases. Then the defect was closed after incising and reflecting the anterior rectus sheath to create a new linea alba. In this process the rectus sheath were sutured in the midline using a continuous polypropylene suture material without tension (as seen in fig.). After that a scaffold of two layered polypropylene darning with a woven pattern was made between the two lateral cut edges of the rectus sheath in which the newly created linea alba was also included (as seen in fig). In this technique, the usage of large quantity of foreign material (polypropylene mesh) is avoided. At the same time, adequate reinforcement is obtained with a woven knit of polypropylene. No mesh is needed as this sutured scaffolding strengthens the whole anterior abdominal wall and thus prevents recurrence. (Fig $1 \& 2$ )

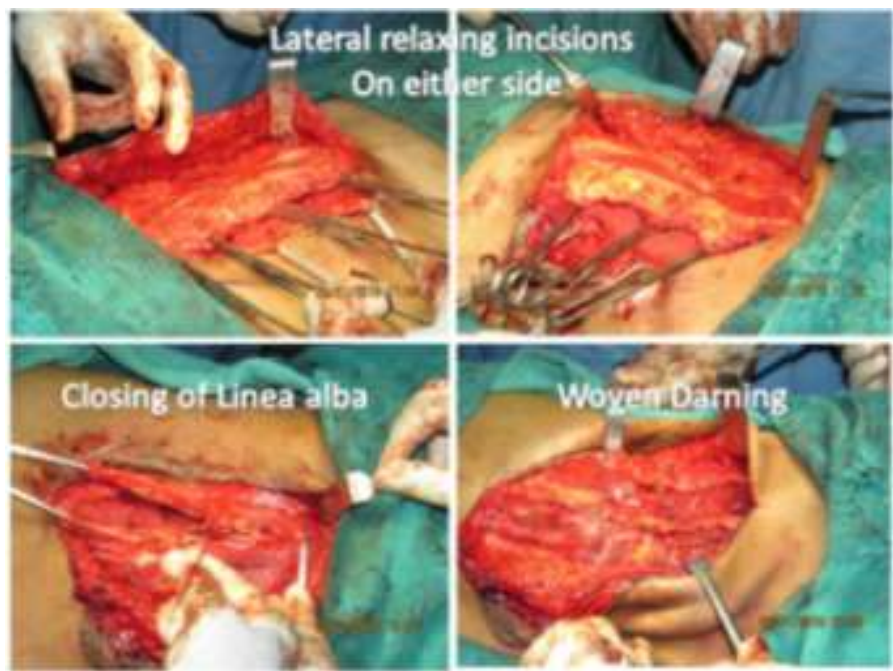

Fig 1: Steps of Woven Darning technique.

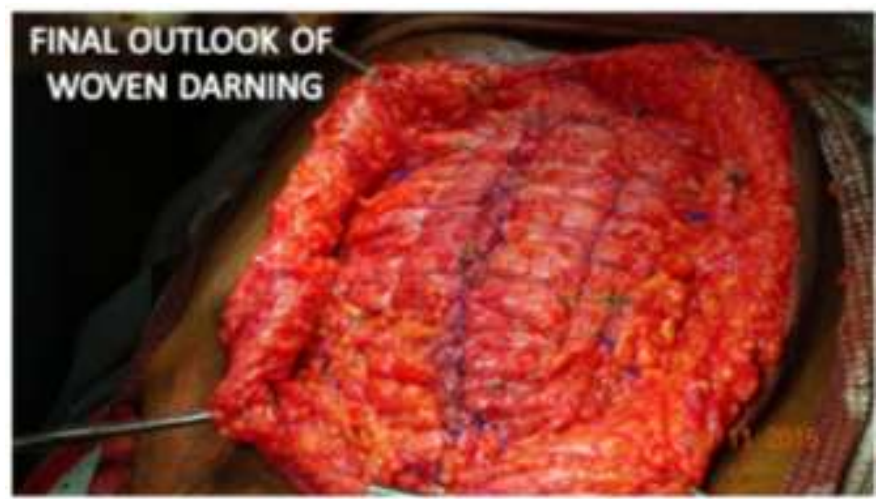

Fig 2: Final outlook

Follow up: Regular follow up done at 15 days, I month, 3 months, 6 months and one year postoperatively.

\section{Results}

Of 20 cases, 12 were males and 8 were females with age preponderance of 15 to 70 years. The average age was 35 years with commonest age group being 27 to 45 years. History of previous surgery followed by wound infection was present in all cases. In females, $62.5 \%$ hernias were due to prior Obstetric and Gynecological procedures. Of 20 cases studied, $30 \%$ defects were composite extending both above and below the umbilicus. In all the cases, the defects were at least more than $5 \mathrm{cms}$ with lateral retraction of the rectus muscles. Demographic profile is enlisted in tables 1,2,3,4. 
TABLE 1: Demographic and History profile:

\begin{tabular}{|l|l|}
\hline Number of Males & 12 \\
\hline Number of Females & 8 \\
\hline Average age in Years & 35 \\
\hline
\end{tabular}

TABLE 2: Previous Surgery:

\begin{tabular}{|l|l|}
\hline Emergency Laparotomy for Perforative peritonitis & 9 \\
\hline Emergency Laparotomy for Intestinal Obstruction & 6 \\
\hline Laparotomy for Obstetric and Gynecological Procedures & 5 \\
\hline
\end{tabular}

TABLE 3: Site of Defect

\begin{tabular}{|l|l|}
\hline Upper Midline & 9 \\
\hline Lower Midline & 5 \\
\hline Combined (both above and below the umbilicus) & 6 \\
\hline
\end{tabular}

TABLE 4: Defect Size

\begin{tabular}{|l|l|}
\hline Defect Size & No of Patients \\
\hline 5 to 7 CMS & 2 \\
\hline 7 to 9 CMS & 5 \\
\hline MORE THAN 9 CMS & 13 \\
\hline
\end{tabular}

TABLE 5: Post-operative Complications:

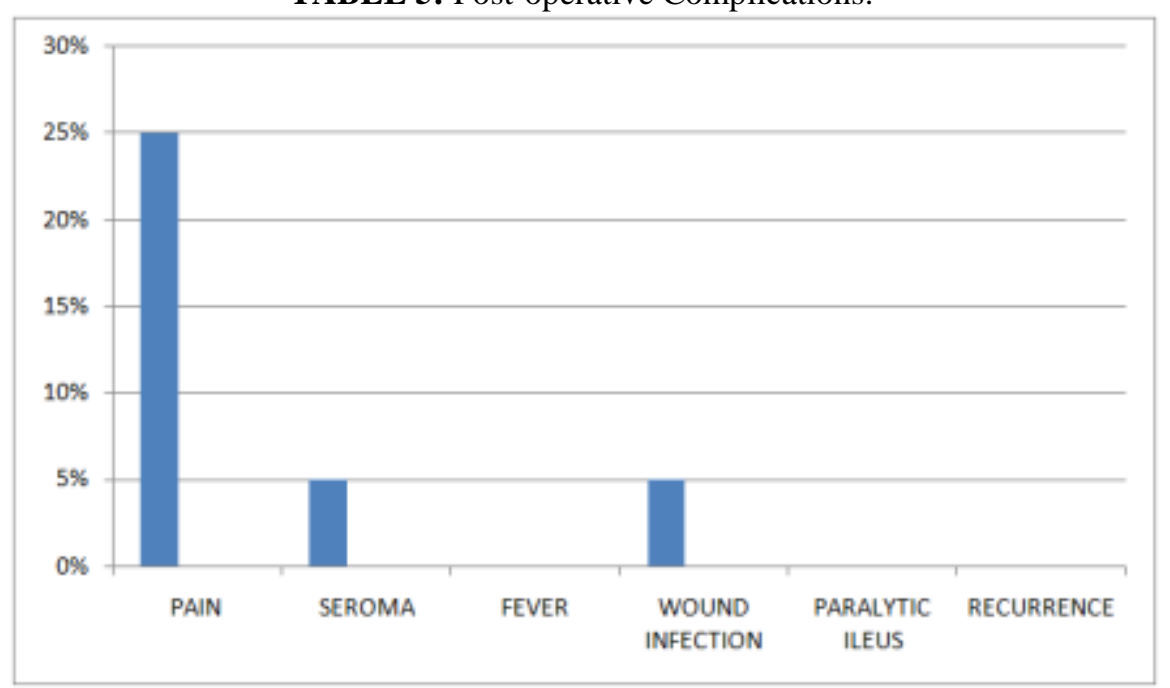

Of the twenty cases studied, there were no undue postoperative complications except for one case that developed serous discharge from wound on second day which got settled in a week time and one patient who had minimal wound infection and all patients were discharged with healthy wound. Postoperative pain was present in $25 \%$ of patients beyond one day. But none had pain beyond four days (Table 5). The duration of postoperative hospital stay ranged from 10 days to 15 days with a mean hospital stay of 12.5 days and mode being 11 days. Only one patient who developed infection had a prolonged stay of 15 days. Regular follow ups were done at 15 days, I month, 3 months, 6 months and one year postoperatively from the time of discharge and it revealed no recurrence in any case.

The result of this study (Technique) emphasis the possibility of a perfect tension free repair of anterior abdominal wall defect without using a polypropylene mesh. At the same time, adequate reinforcement is obtained with no recurrence so far. The avoidance of usage of large quantity of foreign material (polypropylene mesh) eliminates the risk of mesh infection. This technique is an anatomically and functionally sound procedure.

\section{Discussion}

Incisional hernias result as a failure of the fascial tissues to heal following a major abdominal surgery. This condition not only creates psychological trauma to patient in the first instance but also scars the surgeon's name and fame if it recurs after its repair. Since the previous century, a lot of studies have been focused on this particular condition in achieving a tension free repair and prevention of recurrence. Open Anatomical repair, Open mesh repair, Laparoscopic mesh repair [4], Keel's repair and Mayo's double breasting repair etc have been studied extensively but most are followed by high percentage of recurrence and postoperative complications [1]. 
Abrahamson first introduced a new concept of restoring the functional Anatomy of the anterior abdominal by creating a new linea alba with anterior rectus sheath and using the Shoe lace technique to strengthen the anterior abdominal wall [2]. The disadvantage of this technique was because of the fact that in patients suffering from incisional hernia, the abdominal musculature tone was very poor and so when attempting to approximate the recti, the muscles were further weakened due to the tension created [3]. (Fig 3)

\section{TENSION SUTURING IN SHOELACE TECHNIQUE}

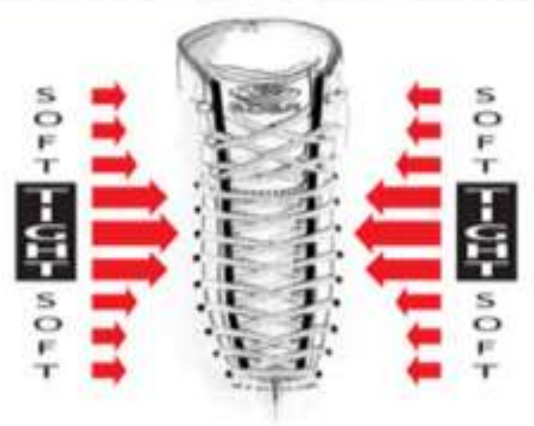

Fig 3: Shoelace technique

So, a modified version of this technique was then introduced by placing a mesh that is closely sutured to the cut lateral edges of the rectus sheath to prevent herniation through the gaps that exist at the lateral edges at the same time reinforcing the anterior abdominal wall [5]. (Fig 4)

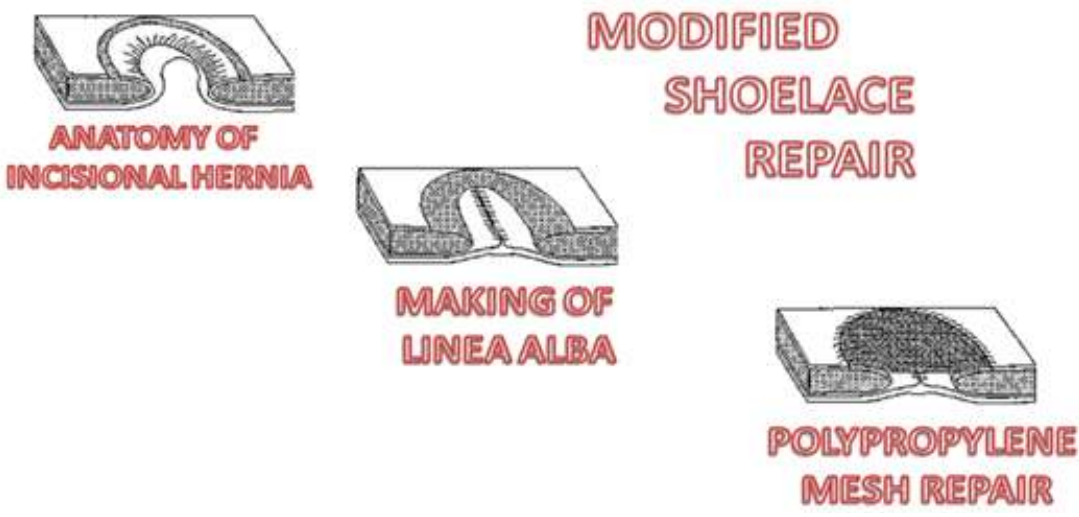

Fig 4: Modified shoelace techniques

A better understanding of applied anatomy of anterior abdominal wall will favor the understanding of incisional hernias. The abdominal wall musculature (Rectus Abdominis) usually tend to shorten and deviate away from midline (linea alba) in normal state of tonic contraction. Linea alba acts as an anchor and prevents this lateral deviation and thus acts as a dynamic girdle in holding back the abdominal contents. Defect in linea alba leads to loss of this anchorage and the muscles deviate laterally while they contract and the midline becomes so weak leading to a bigger defects in incisional hernias. Understanding this applied anatomy prompts the idea of "creation of new linea alba with a reinforcement of the anterior abdominal wall" in repairing ventral hernias repair and to prevent recurrence especially in hernias with large defect.

This concept of "creation of new linea alba with a reinforcement to the anterior abdominal wall" is the key point in our new 'Woven Darning technique'. Where in we incise the anterior rectus sheath at an appropriate site from the midline and raise medial flaps to create the new linea alba. And used a scaffold of two layered polypropylene darning with a woven pattern to bridge the gaps in the rectus sheath and reinforce the anterior abdominal wall. This provides an anchorage to the recti which is on par with the original anatomical dynamic girdle. Now in our technique, it is clear that anterior abdominal wall muscles take up their normal anatomical state and function. So this technique addresses the defect both anatomically and functionally. The postoperative period is uneventful because, the entire repair is extra-peritoneal and so postoperative recovery of the patient is too smooth. 


\section{Conclusion}

By this woven darning technique gives a tension free repair of the Hernia and defects in lateral cut edges of the Rectus sheath are well addressed. Anterior abdominal musculature takes up their normal anatomical position and function. The usage of large quantity of foreign material (polypropylene mesh) is avoided and so a complicated postoperative mesh infection is avoided.

\section{References}

[1]. Loh A. Rajkumar JS,South LM (1992) Anatomical repair of Large Incisional hernias Ann R Coll Surg Engl 74:100-105

[2]. Abrahamson Jack (1997) Epigastric, Umbilical, Ventral hernias. Maingot's Abdominal Surgery 423:430

[3]. Talib A. Majid, Mohanad M. Yassen, Weseem M. Shakir., Shoelace Darn repair of Abdominal Incisional Hernias : vol 6., No. :3., 2007

[4]. David L Sanders, Andrew N. Kingsnorth ., Modern management of Incisional hernias ., BMJ 2012 ., 344 e 2843 doi: 10.1136/bmj.e2843

[5]. M.A. Joshi. M.B. Singh.M.A. Gadhire .,Study of Outcome of Modified Shoelace repair for midline Incisional Hernia : Springer: 2014 